\title{
Relación entre los conceptos: información, conocimiento y valor. Semejanzas y diferencias
}

\author{
Miguel Ángel Rendón Rojas \\ Doctor en Filosofía. Investigador. Centro Universitario de \\ Investigaciones Bibliotecológicas. Universidad Nacional Autónoma \\ de México \\ E-mail: marr@servidor.unam.mx
}

\section{Resumen}

Se analiza el problema de las semejanzas y diferencias entre la información, el conocimiento y el valor tomando como elementos de análisis sus fuentes de origen, las actividades que se realizan para obtenerlas y sus características específicas. Se concluye que existen diferencias esenciales entre esos tres fenómenos, y que no pueden ser vistos como tres etapas de un mismo y único proceso. La misma conclusión es válida para diferenciar la sociedad de la información, la sociedad del conocimiento y la sociedad del valor.

\section{Palabras clave}

Análisis de conceptos; Información; Conocimiento; Valor; Axiología y bibliotecología.

\section{Relation between the concepts of information, knowledge and value. Their similarities and differences}

\begin{abstract}
The problem of the similarities and differences between information, knowledge and value is analyzed, taking into consideration their sources, activities undertaken to obtain them and their specific characteristics as elements of analysis. The author concludes that there are essential differences between those three phenomena and should not be seen as three phases of the same and unique process. The same conclusion is also valid to differentiate Information Society, Knowledge Society and Value Society.
\end{abstract}

\section{Keywords}

Conceptual analysis; Information; Knowledge; Value; Axiology and library science.

\section{INTRODUCCIÓN}

Aunque existen distintas propuestas sobre el objeto de estudio de la bibliotecología, ${ }^{1}$ la información siempre ocupa un lugar central dentro del campo fenoménico de dicha ciencia, y un problema a investigar es la relación que guarda con el conocimiento. Asimismo, tomando en cuenta la distinción entre intelecto y voluntad como dos facultades de la razón, cuyas actividades respectivas son conocer y querer, y el interés por problemas éticos y en general por la responsabilidad social de los bibliotecólogos, ${ }^{2}$ hace que sea importante analizar la relación existente entre la información y el valor, para responder la pregunta cómo y por qué influye la información en el compromiso del ser humano frente a su realidad, e identificar el proceso que ocurre cuando se produce el paso de "tener información" a "tener un valor".

\section{INFORMACIÓN Y CONOCIMIENTO}

Las semejanzas y diferencias entre la información y el conocimiento se analizarán tomando como criterio las fuentes de donde se originan, las actividades que se realizan para obtenerlas y los resultados propiamente dichos.

Fuentes de la información y el conocimiento. Podemos constatar que el material que sirve como punto de partida o materia prima para obtener información es diferente al que se emplea para obtener conocimiento. Ya hemos mencionado que la información tiene como origen datos u objetos sensibles: "La información está más allá de los datos, ella no actúa directamente sobre nuestros órganos de los sentidos, sino que son los datos los que actúan

${ }_{1}$ Algunas propuestas de objetos de estudio de la bibliotecología son la biblioteca, la clasificación, el documento, la información, el fenómeno comunicacional, la actividad bibliotecaria, el sistema de información documental entre otros. Cf. MARTÍNEZ RÍDER, Rosa María y RENDÓN ROJAS, Miguel Ángel. "Algunas propuestas latinoamericanas de objeto de estudio para la investigación bibliotecológica”. En Revista Interamericana de Bibliotecología. V. 27, N. 1. Enero-junio de 2004. Medellín, Colombia: Universidad de Antioquia, Escuela Interamericana de Bibliotecología, 2004. pp. $13-44$.

${ }^{2}$ GÓMEZ-PANTOJA FERNÁNDEZ, Aurora. "Bases teóricas para el desarrollo de un código de Ética para la profesión bibliotecaria”. En Boletín de la ANABAD. N. 3. Julio-septiembre 2001. Madrid: ANABAD, 2001. pp. 15-35. PÉREZ PULIDO, Margarita. "Códigos de ética de los bibliotecarios y otros profesionales de la información: comentario y análisis comparativo". En Boletín de la ANABAD. N. 3. Julio-septiembre 2001. Madrid: ANABAD, 2001. pp. 37-57. 
sobre estos últimos". ${ }^{3}$ Por tanto la información no existe como un ente acabado y autónomo, sino que es construida a partir del mundo material, y existe como cualidad secundaria de un objeto particular: del signo lingüístico registrado ${ }^{4}$.

Por su parte el conocimiento tiene como fuente la información misma, por lo que es un producto posterior y surge a partir de ella. Si la información se elabora a partir de objetos materiales para después aparecer como ente ideal; el conocimiento retoma ese ente ideal para construirse. Una distinción semejante la encontramos en Shera, quien expresa: "distinguimos entre información y conocimiento [...] la información es el insumo del conocimiento, y siempre es recibida a través de los sentidos [...] no puede haber conocimiento sin un conocedor. $^{5}$

Actividades para construir la información y el conocimiento. También las actividades por las cuales aparecen los fenómenos analizados son diferentes. Para que se obtenga información es necesario realizar una síntesis, entendida ésta desde una perspectiva kantiana como unión de dos extremos opuestos. Dicha síntesis se lleva a cabo por una acción del sujeto que une elementos objetivos (datos y estímulos sensoriales) y subjetivos (estructuras interpretativas del sujeto). Estos últimos sirven para procesar, organizar, estructurar y dar forma a los datos, lo que permite extraer las cualidades secundarias presentes potencialmente en los símbolos, esto es, interpretarlos, enlazándolos con su referente y sentidos ideales. De esta manera se comprende la etimología de la palabra información que proviene de informar, que en latín es informare que significa literalmente "dar forma", ${ }^{6}$ esto es, establecer límites a la materia, dotarla de estructura y organización.

\footnotetext{
${ }^{3}$ RENDÓN ROJAS, Miguel Ángel. Bases teóricas y filosóficas de la bibliotecología. México: CUIB, UNAM, 1998. p. 62.

${ }^{4}$ Según Locke las cualidades primarias son inseparables de los cuerpos y se captan de manera inmediata como son la extensión o la figura; las cualidades secundarias no están presentes de manera actual en los cuerpos, sino que aparecen a posteriori gracias a la percepción de un sujeto, como por ejemplo los colores, los sonidos y los sabores.

${ }^{5}$ SHERA, Jesse H. Los fundamentos de la educación bibliotecológica. México: UNAM/CUIB, 1990. p. 118. La semejanza con nuestro pensamiento es sólo en lo relativo a que la información es insumo del conocimiento, pero existe una diferencia en cuanto que para nosotros la información no se capta por los sentidos.

${ }^{6}$ GÓMEZ DE SILVA, Guido. Breve diccionario etimológico de la lengua española. México: El Colegio de México, Fondo de Cultura Económica, 1988. p. 377.
}

El problema sobre la objetividad de la información que surge porque ésta es el resultado de una actividad del sujeto, y por ende puede parecer subjetiva, se resuelve satisfactoriamente desde dos ángulos. En primer lugar "la objetividad de la información viene dada por los datos que existen independientemente del sujeto [...] los podemos diferenciar de otros datos, de alucinaciones". En segundo lugar las estructuras que se utilizan para la organización y significación del material empírico también tienen su valor objetivo, porque rechazamos la idea de que dichas estructuras sean inventadas arbitrariamente. Las estructuras mencionadas son objetivas porque son el resultado de un proceso psicogenético que tiene su base en la naturaleza biológica y psicológica del ser humano, ${ }^{8}$ aunque al mismo tiempo dicho proceso se ve inmerso en contextos sociohistórico-culturales determinados. Así es que cada sociedad instituye sus esquemas para interpretar los símbolos con base en las relaciones que el ser humano establece con la naturaleza y con otros seres humanos en el proceso de producción material, espiritual y biológico. Es evidente que las mencionadas relaciones e interrelaciones son objetivas porque permiten la existencia misma de la especie humana y posibilitan el proceso de imposición de las voces, ${ }^{9}$ o lo que U. Eco denomina "convención cultural"10.

Ahora bien, para que se produzca el conocimiento es indispensable realizar no sólo la estructuración e interpretación de símbolos, sino otra serie de actividades más complejas. Es indispensable no sólo la decodificación de símbolos, sino la memorización, el análisis, que permite identificar los elementos constituyentes de lo que se está conociendo y sus relaciones como partes del todo; la síntesis en la cual se vuelven a reunir en un todo aquello que se fragmentó y descubrir las relaciones del todo con las partes; tener una visión dialéctica que permita descubrir la interconexión de la información nueva con otras informaciones y conocimientos del sujeto cognoscente; elaborar inferencias de varios tipos como

${ }^{7}$ RENDÓN ROJAS, Miguel Ángel. Op. Cit. p. 64.

${ }^{8}$ Cf. PIAGET, Jean. "Lógica formal y psicología genética". En PIAGET, J. Estudios sobre lógica y psicología. Madrid: Alianza Editorial, 1982. pp. 149-175. PIAGET, J. El nacimiento de la inteligencia en el niño. México: Grijalvo, 1990. PIAGET, J. Estudios de Psicología genética. Buenos Aires: Emece, 1973.

${ }^{9} \mathrm{Cf}$. BEUCHOT, Mauricio. La filosofía del lenguaje en la Edad Media. 2 ${ }^{\mathrm{a}}$ ed. México: UNAM, Instituto de Investigaciones Filológicas, 1991. p. 50 .

${ }^{10}$ ECO, U. Tratado de Semiótica general. Barcelona: Ed. Lumen, 1988. p. 42. 


\section{Miguel Ángel Rendón Rojas}

inferencias deductivas ${ }^{11}$, inductivas, ${ }^{12}$ abductivas $^{13} \mathrm{y}$ hermenéuticas; ${ }^{14}$ aplicar las nuevas ideas y visiones del mundo obtenidas; evaluar todo el proceso y asimilar el constructo cognoscitivo obtenido. Asimismo es necesario subrayar que para realizar los pasos antes mencionados es indispensable utilizar no sólo las capacidades intelectuales puras, sino también emplear la fantasía, la imaginación y la creatividad. ${ }^{15}$

En otras palabras, utilizando el pensamiento de Piaget, el conocimiento es construido por el sujeto con base en la asimilación, integración y reorganización de estructuras que le permiten interpretar el mundo e interactuar con él. ${ }^{16}$ Dichos esquemas son una serie de operaciones susceptibles de realizarse en situaciones semejantes, no necesariamente idénticas, que le permiten resolver problemas e interrelacionarse con su entorno. Pero cuando se presenta una situación nueva no se pueden aplicar los esquemas disponibles, por lo que se debe crear un nuevo esquema modificando o combinando esquemas anteriores. ${ }^{17}$ De esta manera, el conocimiento es crear y re-crear sentidos, construir y re-construir ideas, formar y re-formar juicios, producir y re-producir teorías, fundamentar y re-fundamentar discursos, elaborar y reelaborar visiones del mundo.

Desde el punto de vista filosófico el elemento creativo y de innovación presente en el conocer es posible gracias a que, como lo expresara P. Ricoeur, la interpretación no se realiza de manera reduccionista, esto es, no se busca una relación unívoca entre símbolo y referente como lo haría una máquina con ayuda de un programa predeterminado

${ }^{11}$ Las inferencias deductivas tienen lugar cuando existe la relación de consecuencia lógica entre un juicio o enunciado, llamado conclusión, y otros juicios o enunciados, llamados premisas. La consecuencia lógica se da cuando es imposible que las premisas sean verdaderas y al mismo tiempo la conclusión sea falsa, esto es, si las premisas son verdaderas, necesariamente la conclusión será verdadera.

${ }^{12}$ Las inferencias inductivas consisten en obtener una conclusión con base en generalizaciones de casos particulares. Su estructura general es: $a_{1}$ es $P, a_{2}$ es $P, a_{3}$ es $P, \ldots, a_{n}$ es $P$, por lo tanto toda $a$ es $P$. No hay consecuencia lógica porque nada impida que la siguiente $a_{n+1}$ no sea $P$, convirtiendo a la conclusión en falsa, por lo que se habla sólo de verosimilitud en los razonamientos inductivos.

${ }^{13}$ La abducción o inferencia hipotética fue propuesta por Peirce y es una conjetura o hipótesis que se propone para resolver un problema teórico. Cf. FANN, K. T. Peirce's Theory of Abduction. The Hague, Martinus Nijhoff, 1970. MAGNANI, Lorenzo. Abduction, reason, and science: processes of discovery and explanation. York: Kluwer Academic/ Plenum, 2001.

${ }^{14}$ Las inferencias hermenéuticas consisten en encontrar nuevos sentidos y significados, gracias a un horizonte hermenéutico más amplio que permite "contemplar" la realidad desde otra perspectiva. ${ }^{15}$ Cf. TERRICABRAS, Josep-Maria. Atrévete a pensar: la utilidad del pensamiento riguroso en la vida cotidiana. Barcelona: Paidos, 1999.

16 PIAGET, Jean. La epistemología genética. Madrid: Debate, 1986. p. 154-155.

${ }^{17}$ Ibidem. pp. 41-90. y del cual no se podría salir, sino se hace de una manera más abierta, donde es posible encontrar una referencia secundaria o desdoblada. Pero al mismo tiempo dicha referencia desdoblada (nuevo conocimiento) no es una ficción o un falso logos porque sus raíces se encuentran enclavadas en el Ser. La facultad de construir y reconstruir nuevas referencias desdobladas descansa en la capacidad simbólica del ser humano descubierta por Cassirer en su análisis de la comprensión, en donde da cuenta de que el Ser aparece en forma simbólica al hombre.

En efecto, Cassirer descubre que el hombre no se enfrenta directamente con la naturaleza, sino que tiene una relación mediata con ella a través de un sistema intermedio entre el mundo humano y el mundo natural que conecta esas realidades. Dicho sistema está constituido por las formas simbólicas, que son las formas fundamentales de la comprensión del mundo. Lo anterior se debe a que el mundo natural no posee sentido ni significado, sino simplemente es, y son las formas simbólicas las que le otorgan sentidos, porque permiten valorar lo que el ser humano experimenta como real y entender la realidad al fijar significados a lo que se percibe. Así pues es el ser humano quien configura su mundo por medio de la actividad simbólica. Ahora bien, debido a que los símbolos son signos convencionales porque su función significativa no es resultado inmediato de la relación del símbolo con su referencia, como lo es la relación causa-efecto del signo-índice o imagensemejanza del signo-icono con el referente, entonces detrás de cualquier referencia siempre es posible hallar una expresión subjetiva, lo que deja la puerta abierta a la mencionada construcción de una referencia desdoblada y de nuevos conocimientos.

Si se define el conocimiento como una creencia verdadera y justificada ${ }^{18}$, donde por creencia se entiende un pensamiento aceptado y que se afirma sin temor a equivocarse; por verdad, una característica del enunciado que consiste en que hay una correspondencia entre lo que se dice y la realidad ${ }^{19}$, y por justificación, el proporcionar las razones por las que se tiene esa creencia; entonces podemos concluir que las actividades mencionadas para obtener conocimiento proporcionan la justificación y al mismo tiempo dan la oportunidad de obtener la novedad en el conocimiento y la asimilación del mismo.

18 VILlORO, Luis. Creer, saber, conocer. México: Siglo XXI, 1996.

19 "Decir del no ser que es y decir del ser que no es, es falso. Decir del no ser que no es y del ser que es, es verdadero". ARISTÓTELES. Aristotelis metaphysica. Metafísica de Aristóteles. Ed. trilingue por Valentín García Yebra. Madrid: Gredos, 1982. 1011b. 
Como ilustración de las actividades que se realizan para obtener información primero y conocimiento después, ponemos como ejemplo una gráfica de la distribución ocupacional de la población económicamente activa (PEA) de un país (figura 1).

Para obtener información de esa gráfica se descifran sus datos, se entiende que la coordenada vertical indica el número de personas en edad económicamente activa, y que en la coordenada horizontal se representan el tipo de actividades económicas agrupadas por sectores; asimismo se interpreta la altura de las barras como el número de individuos que se encuentran en un sector, y consecuentemente se pueden comparar las barras y observar cuál es mayor y cuál es menor. De esta manera se obtiene la información del número de personas que laboran en cada sector de la economía y de desempleados. Hasta ese momento, se ha obtenido únicamente la información de lo que nos "dice" la gráfica.

Si se desea obtener conocimiento, además es necesario analizar la metodología que se utilizó, las técnicas de recolección de datos utilizadas, evaluar el grado de confiabilidad del estudio, qué edades se determinaron como límites de la PEA, cómo se midió el desempleo. Al mismo tiempo, se deben manejar los porcentajes de los que se está hablando, para así saber si esos números absolutos son muchos o pocos en relación con el todo. Se puede calcular el porcentaje de la PEA en relación con la población total y saber cuantas personas dependen económicamente de un trabajador. Asimismo se deben comparar esos datos con los de otros años para determinar la dinámica de la economía, identificar tendencias y realizar proyecciones. Por otro lado esa información debe ser comparada con los datos de otros países, relacionar los datos obtenidos con conocimientos de la teoría política, la historia, la filosofía, la geografía, la economía, la sociología, entre otras, para comprender ciertos fenómenos como la migración, la criminalidad, la pobreza, la desigualdad social y económica, los movimientos sociales, la demagogia, el lenguaje político, la publicidad, los valores sociales y culturales, etcétera.

Naturaleza de la información y el conocimiento. Aunque ambos objetos son entes ideales, sin embargo, mientras la información es un ente ideal objetivado ${ }^{20}$, el conocimiento es un ente ideal subjetivado ${ }^{21}$, es decir, el

\footnotetext{
${ }^{20}$ RENDÓN ROJAS, Miguel Ángel. "La información como ente ideal objetivizado". En Investigación bibliotecológica: Archivonomía, Bibliotecología e Información. México: UNAM-CUIB, 1995. V. 9, N. 18. Enero-junio 1995. pp. 17-24.

${ }^{21}$ Shera utiliza una expresión de Helprin, donde dice: "El conocimiento es información subjetiva”. SHERA, J. Op. cit. p. 128.
}

\section{FIGURA 1}

Distribución ocupacional de la PEA del país por sectores económicos. (Los datos son ficticios y sólo fueron concebidos para ejemplificar nuestra posición teórica)

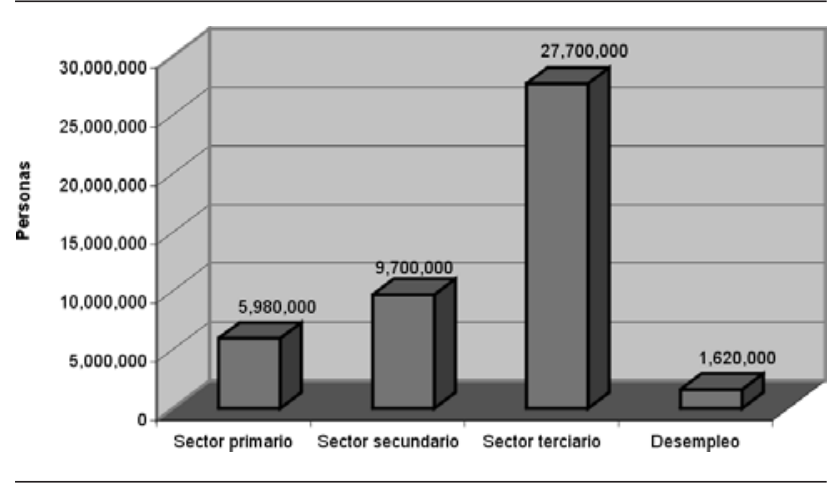

conocimiento existe en el sujeto y sólo en el sujeto, al salir de éste se convierte en información. De lo anterior se sigue como corolario dos tesis. La primera consiste en que se niega la posibilidad de la existencia del tercer mundo popperiano, el mundo de la ciencia. Éste es un mundo construido por sujetos (científicos), como conocimiento se encuentra en los sujetos, no es una substancia en sí, sino que necesita un sustrato para subsistir, y por consiguiente no puede evolucionar o actuar independientemente de los sujetos. La segunda tesis es que el profesional de la información, al trabajar con documentos, trabaja con información objetivada y no con conocimientos. Esto es importante porque permite distinguir las funciones de los profesionales de la información y de la educación. El primero puede ayudar al usuario a obtener conocimiento a partir de la información obtenida a través de los documentos, pero ésa no es su función esencial, porque ésta es ayudar al usuario a acceder al mundo de la información documental.

Finalmente la distinción entre información y conocimiento nos permite diferenciar el flujo de la información, el ciclo social de la ésta y el proceso de aprendizaje. El primero consiste en el "conjunto de documentos publicados y los documentos científicos no publicados [...] que aparecen constantemente y son utilizados en la práctica histórico-social con la finalidad de intercambiar información científico-técnica". ${ }^{22}$ Por su parte Mijaílov afirma que la informática estudia el

\footnotetext{
22 GORKOVA, V. I. y T. I. Gusieva. "Análisis de los flujos de documentación e información y estudio de las solicitudes de los usuarios de información". En Seminario. Introducción a la Informetría. Análisis de los flujos informacionales y evaluación de las fuentes de información. Melvyn Morales Morejón. Comp. La Habana: IDIT/SOCICT-CI. pp. 25-36. p. 27.
} 
ciclo social de la información, que incluye la generación, recolección, procesamiento analítico-sintético, almacenamiento, búsqueda y recuperación, diseminación y uso de la información, que a su vez lleva de nuevo a la generación de información, repitiéndose de esa manera el ciclo. ${ }^{23}$

\section{FIGURA 2}

Ciclo social de la información según Mijaílov, Chernii y Guiliarevskii ${ }^{24}$

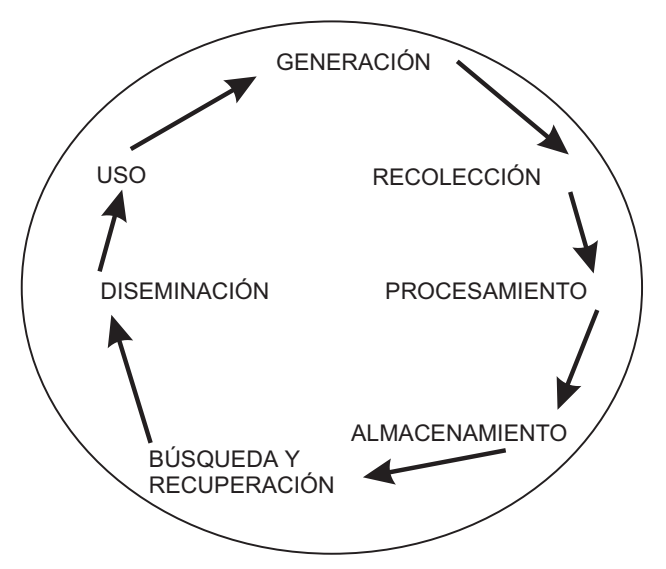

Por su parte, el proceso de aprendizaje grosso modo se puede entender desde el enfoque cognoscitivo como la construcción del conocimiento a través de la información que se recibe. De esta manera el maestro estrictamente hablando no proporciona conocimiento a sus alumnos, sino que son estos últimos quienes lo elaboran a partir de la información que les proporciona el maestro y los libros.

Se puede representar el ciclo de la informaciónconocimiento de la siguiente manera (figura 3):

Es posible descubrir que en determinada etapa los tres procesos mencionados coinciden, pero no son equivalentes porque después se separan. El flujo de información documental incluye todos los documentos que se utilizan para intercambiar información, los cuales siguen un ciclo social que va de su generación a su uso, y el paso de la información al conocimiento incluye en su proceso al flujo y al ciclo social de la información (figura 4).

${ }^{23}$ MIJAILOV, A. I.; A. I. Chernii y R. S. Guiliarevskii. Fundamentos de la informática. Moscú, La Habana: Nauka, Academia de Ciencias de Cuba, Instituto de Documentación e Información Científica y Técnica. T. 1. 1973. p. 59, 60.

${ }^{24}$ Cf. GORBEA PORTAL, Salvador. Producción y comunicación científica latinoamericana en ciencias bibliotecológica y de la información. Tesis Doctoral. Universidad Carlos III de Madrid, Facultad de Humanidades, Comunicación y Documentación. Getafe, 2004. p. 22.
FIGURA 3

Ciclo información-conocimiento

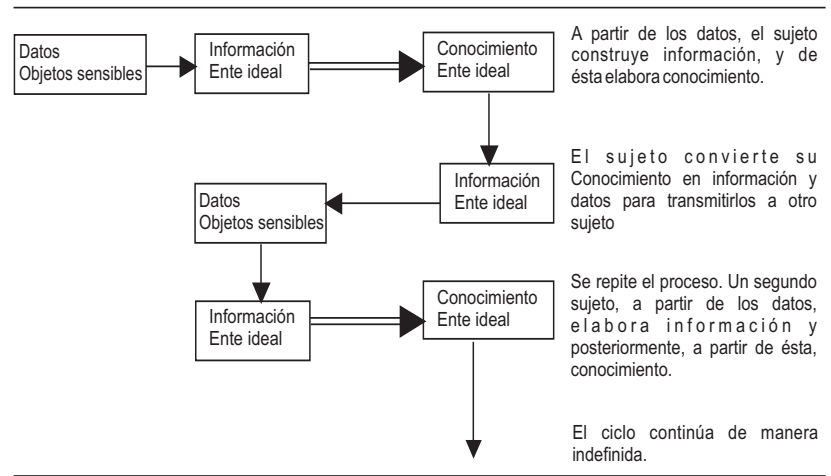

FIGURA 4

Relación entre el flujo de información documental, el ciclo social de la información y el proceso informaciónconocimiento

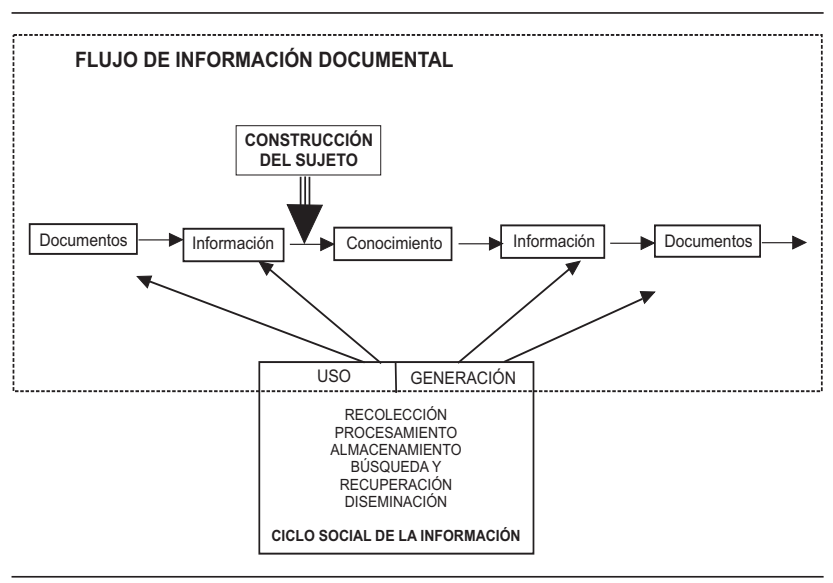

Finalmente, la distinción entre información y conocimiento nos ayuda a esclarecer la diferencia entre sociedad de la información y del conocimiento. Al igual que el conocimiento es posterior y superior a la información, se puede concluir que la sociedad del conocimiento es superior y posterior a la sociedad de la información. Si definimos a la sociedad de la información como "el conjunto de relaciones sociales en un espacio social (institucionalidad) altamente dinámico, abierto, globalizado y tecnologizado, que se apoyan y realizan a través de la información; la cual es igualmente dinámica, abierta, globalizada, tecnologizada además de mercantilizada", ${ }^{25}$ entonces la sociedad del conocimiento será el conjunto de relaciones sociales en un espacio social (institucionalidad) altamente tecnologizado que por un lado funciona gracias al conocimiento objetivado en instrumentos, y por otro, debido a que el conocimiento

${ }^{25}$ RENDÓN ROJAS, Miguel Ángel. "Un análisis del concepto sociedad de la información desde el enfoque histórico". En Información, cultura y sociedad. Buenos Aires: Universidad de Buenos Aires. Instituto de Investigaciones Bibliotecológicas. N. 4/2001. pp. 9-22. p. 16. 
existe en el sujeto, esas relaciones sociales se dan entre sujetos con conocimiento, entendido éste no sólo como un saber referido al cómo hacer, sino al qué y por qué de la realidad. Mientras no se tenga claro lo anterior existe el peligro de que al hablar de sociedad del conocimiento se haga como slogan mercadotécnico para hacer atractivos ciertos productos que lanzan empresas transnacionales y consecuentemente contiene un elemento ideológico implícito. ${ }^{26}$

\section{INFORMACIÓN, CONOCIMIENTO Y VALOR}

Pasemos ahora a estudiar la relación existente entre la información y el conocimiento con el valor. Para empezar diremos que concebimos el valor como un objeto, en su acepción más amplia -todo lo que es nombrado -, hacia el que tiende una relación volitiva del sujeto con el fin de "poseerlo" para disfrutarlo porque es apetecible, y sirve para comprender, orientarse e interactuar con la realidad. En su realidad ontológica más abstracta se presenta como objeto: la verdad, la bondad, la belleza, por ejemplo; y en su manifestación ontológica concreta, como cualidad de algo. Así por ejemplo la verdad (falsedad) aparece como propiedad de los enunciados o proposiciones, por lo que se puede afirmar que el enunciado "los metales conducen electricidad" es verdadero.

$\mathrm{Al}$ analizar los valores desde el punto de vista filosófico se han formulado dos tipos de respuestas sobre su status ontológico. La teoría platónica del valor que lo concibe como algo existente de manera absoluta e independiente de las cosas, y por lo tanto debe de ser descubierto, y la teoría nominalista de los valores que afirma que el valor está fundamentado en el agrado o desagrado que causa una cosa, siendo en el acto del juicio valorativo cuando se crean los valores. Nuestra posición está basada en una visión dialéctica que al mismo tiempo reconoce la objetividad del valor dada por el momento ontológico, esto es, el ser fundamenta el valor; y la subjetividad del mismo dada por la actividad del sujeto que en el proceso de su formación anthropo-ontológico va construyendo históricamente los valores.

Como ya se mencionó, el mundo natural no posee sentido ni significado, pero el ser humano no puede orientarse en un mundo sin sentido, por lo que siempre busca y construye el sentido del mundo. Ahora bien, esa cons-

\footnotetext{
${ }^{26}$ Cf. RENDÓN ROJAS, Miguel Ángel. "Sociedad del conocimiento". En ALMADA DE ASCENCIO, Margarita; Juan José Calva González; Patricia Hernández Salazar; Catalina Naumis Peña y Miguel Ángel Rendón Rojas, ed. Contribución al desarrollo de la Sociedad del Conocimiento. México: UNAM, CUIB. pp. 50-57.
}

trucción de sentidos y valores no es arbitraria, derivada de posiciones individualistas o grupales cerradas, sino que son apariciones del ser y construcciones del sujeto en su juego ontológico para formarse. Como Gadamer reconoce: el ser humano se caracteriza porque es un ente no acabado, un ser que necesita formarse: " $<<[\ldots]$ no es por naturaleza lo que debe ser $>>$; por eso necesita de la formación." ${ }^{27}$ Ese proceso de formación se lleva a cabo a través del juego hermenéutico, el cual consiste en ir construyendo sentidos y valores dentro de un marco limitado por las reglas del juego. Ahora bien, las reglas no pueden ser propuestas por un agente externo, porque, "la formación no conoce objetivos que le sean exteriores"; ${ }^{28}$ ni tampoco pueden ser establecidas por sujetos individuales porque se rompería con la apertura a la comunidad que exige la formación: "no existe el juego en solitario $[\ldots]$ siempre tiene que haber algún $<<$ otro $>>$."29 Por lo tanto las reglas del juego se deben buscar dentro del mismo juego, éstas se construyen dentro de él. Es en el juego donde el hombre se encuentra con el Ser.

Al mismo tiempo el universalismo-particularismo de los valores se da gracias, el primero a la acción del ser que se des-vela, hecho que Heidegger denomina aletheia; y el segundo a la actividad del hombre, que en su contexto histórico concreta, va moldeando ese ser agregándole sentidos y valores mediante el lenguaje, obras de arte, conocimientos, creencias, axiologías, mitos, religión, filosofías, etcétera. Así pues, los valores son una cierta forma de epifanía del ser revestido de prendas dadas por el sujeto. Una manera de identificar la existencia de un valor a pesar de la multiplicidad histórica y cultural es tratar de responder a la pregunta si ese objeto deseado permite el desarrollo del ser del hombre, entendiendo que ese ser no es individual sino al mismo tiempo comunitario.

Ahora bien, pasando a la relación que nos ocupa encontramos que varios pensadores se han ocupado de ella y realizado diferentes aportaciones. Una de ellas, que podemos calificar como una visión secuencial entre información-conocimiento-valor, la podemos encontrar en Sócrates y los filósofos de la Ilustración quienes identificaban la maldad con el error o la ignorancia. De esta forma, afirmaban que una persona ilustrada y que en sus acciones seguía a la razón no era capaz de realizar actos malos. Así pues, el remedio para aliviar los males morales y sociales consiste en dejarse regir por la razón:

\footnotetext{
${ }^{27}$ GADAMER, Hans-Georg. Verdad y método I. Fundamentos de una hermenéutica filosófica. Salamanca: Sígueme, 1993. p. 41.

${ }^{28}$ Ibídem. p. 40.

${ }^{29} \mathrm{Ibídem}$. p. 150-151.
} 


\section{Miguel Ángel Rendón Rojas}

"El gran medio de disminuir el número de los maniáticos, si quedan, es someter esta enfermedad del espíritu al régimen de la razón." 30

En otra línea del pensamiento, para Kant, que es considerado la culminación del pensamiento ilustrado, la razón práctica es la que orienta a la razón teórica e incluso sobrepasa los límites de ésta porque ella sólo conoce los fenómenos pero no "la cosa en sî" (noúmeno). Sin embargo, la razón práctica sí puede adentrarse al conocimiento del noúmeno y moverse en el reino de la libertad. $^{31}$

Al mismo tiempo, Kant reconoce que la voluntad es esencialmente buena y la maldad sólo es consecuencia de una voluntad débil que no posee la fortaleza necesaria para seguir el deber. El carácter, que es una característica de la voluntad, es definido por Kant como "los firmes designios para querer hacer algo, y [...] la ejecución real de los mismos". ${ }^{32}$ De este modo, la tarea para lograr individuos que hagan el bien consiste en fortalecer la voluntad. Así pues, dentro de esa visión no existe una relación directa entre información y valor, sino mediatizada por una voluntad autónoma debidamente disciplinada en la que el querer y el deber coinciden.

Sin embargo la experiencia histórica de la modernidad, y principalmente del siglo pasado, con dos guerras mundiales, campos de concentración, tecnología de exterminio sofisticada, ha demostrado la incapacidad de la razón para construir el reino de armonía y bienestar prometido por esa visión optimista de la razón. La causa del mal no se encuentra por tanto en la ignorancia, ya los estoicos formularon el aforismo "veo y apruebo lo mejor, hago lo peor", es decir, se puede conocer perfectamente el mal y sin embargo se elige éste. Al respecto, Dostoievsky escribió: "es claro y evidente que el mal se esconde más profundamente de lo que suponen los médicos-socialistas, que en ningún sistema se acabará con el mal, que el alma humana no cambia, que la anormalidad y el pecado nacen de ella misma." 33

Al mismo tiempo, tampoco el mal es la expresión de una voluntad débil, porque hemos sido testigos de acciones que necesitaron de sacrificios y privaciones, que tuvieron

30 VOLTAIRE. Tratado sobre la tolerancia. Madrid: Santillana, 1997. p. 29.

${ }^{31}$ KANT, Inmanuel. Crítica de la razón práctica. México: UAMI, M. A. Porrúa, 2001.

32 KANT, Inmanuel. Pedagogía. Madrid: Akal, 1983. p. 80-81.

33 DOSTOIEVSKY, Fiódor. Mijaílovich. Dnievnik pisatelia (Diario del escritor). En Dostoievsky o russkoi literaturi (Dostoievsky sobre la literatura rusa). Moscú, 1987. p. 267. que enfrentarse a diversos obstáculos y sin embargo se realizaron porque estaban respaldadas por una voluntad de acero, ${ }^{34}$ pero que finalmente eran acciones orientadas por valores que promovían la destrucción de los demás. Una vez más, si recurrimos a las ideas del genial escritor ruso autor de Crimen y castigo, descubrimos que sus héroes, como Raskólnikov, Iván Karamásov o Stavroguin, poseen una voluntad que es fuerte, incluso más fuerte que su sensualidad, pero es una voluntad mala.

La clave de la formación de valores está en la libertad humana, cada ser humano concreto re-crea los valores que las generaciones mayores van presentando. En las sociedades abiertas, donde la libertad en sí misma es un valor, el peligro de que los valores que se transmiten no sean adoptados por las nuevas generaciones siempre está latente, ya que si en la esfera epistemológica podemos decir que sólo puede existir la verdad si existe la posibilidad de la falsedad, "sólo se puede tener razón si se corre el riesgo de equivocarse ${ }^{35}$; en la esfera axiológica, sólo puede existir el valor tomado como propio, si existe la posibilidad de desecharlo. Para la transmisión eficaz de los valores es necesario contar con la colaboración, apertura, casi complicidad de aquellos a quienes se quiere que lleguen los valores, porque los sujetos, en uso de su libertad, siempre tendrán la posibilidad de optar por valores diferentes.

Se habla de transmisión y no de enseñanza de valores porque éstos se comunican a través del ejemplo en la vida misma, no en un salón de clases, más que enseñanza se trata de una comunicación personal y existencial a través del diálogo. En la enseñanza de informaciones o conocimientos el aprendizaje queda asegurado si el estudiante es capaz de repetir y comprender las ideas recibidas. Se puede pedir que se aprendan y repitan principios como "el respeto y la tolerancia son la base de la convivencia social", pero si se tiene la finalidad de que esas frases se conviertan en principios de acción, entonces además de la memorización se debe buscar y estimular la crítica, la reflexión, y sobre todo la aplicación de esos principios, ya que un principio que no se aplique simplemente no es un valor.

Para que suceda lo anterior es indispensable que el que presenta la información cuente con autoridad moral, respeto y cierta sintonía afectiva para entablar una comunicación axiológica con quien debe adoptar el valor. Si se llega a establecer esa empatía existencial, donde las

\footnotetext{
${ }^{34}$ Es sintomático que por ejemplo el creador del Gulag se haya hecho llamar Stalin, que proviene de la palabra rusa stal, que significa acero. 35 FERRARIS, Mauricio. La hermenéutica. México: Taurus, 2001. p. 51.
} 
actitudes y disposiciones del trasmisor del valor llegan a ser vistas como merecedoras de ser incorporadas al proyecto existencial como instrumentos de interpretación de vida, entonces se puede hablar de transmisión de valores. Para que completamente se cierre el ciclo es necesario que se realice una asimilación de esos valores, es decir, su interiorización e integración consciente y crítica a la propia visión del mundo, y un acto de la voluntad que decida hacer propios esos valores.

Así pues, si retomamos los niveles de análisis empleados en la distinción de la información con el conocimiento, podemos decir que la fuente de los valores son la información y el conocimiento que se presentan como objetos paradigmáticos, la actividad que se realiza para dar origen a los valores es una acción de la voluntad que decide hacer propio ese principio que regirá la conducta, y el resultado final es el valor propiamente dicho, como ente ideal, que ayuda a comprender el mundo, y a actuar en él.

Finalmente, esta distinción nos ayudará a comprender porqué una sociedad de la información o del conocimiento no conduce automáticamente a una sociedad justa y democrática que hemos denominado, siguiendo la analogía de los términos "sociedad de la información" y "sociedad del conocimiento", como "sociedad del valor".

$\mathrm{Al}$ analizar los rasgos específicos que caracterizan a la democracia, algunos autores resaltan la combinación de elementos ideales o valores y de propiedades capaces de ser verificadas empíricamente y cuantificables. ${ }^{36}$ Así pues, por un lado la democracia es un proceso, donde el YO reconoce la presencia del otro (alter) con quien se dialoga en condiciones de igualdad, sobre cuestiones que afectan a intereses comunes para llegar a consensos; y por otro lado, la democracia construye los mecanismos necesarios, instituciones sociales, para obstaculizar o castigar las elecciones que prefieren el no-diálogo y optan por el dominio y manipulación, surgiendo de esta manera la democracia como procedimiento.

De esta manera para que se dé el aspecto valorativo es necesario realizar una elección, por lo que se ingresa a la esfera ética-axiológica, donde lo más importante no es el aspecto cognoscitivo, sino el aspecto volitivo. La acción,

${ }^{36}$ Cfr. SARTORI, G. "Democrazia e definizioni", Bologna: Il Molino, 1969. Cap. V. También véase: CANSINO, César. "La democracia entre utopía y realidad". En Universidad de México. N. 540, enero de 1996. México: UNAM, 1996. pp. 66-68. RENDÓN ROJAS, Miguel Ángel. "Democracia: Identidad y diferencia". En Metapolítica. V. 1. N. 2. Abril-Junio de 1997. pp. 277-282. México: BUAP, CEPCOM, 1997. que es condición para la democracia, es una acción moral, es decir, una elección libre y consciente. Sin embargo, la misma posibilidad de la libre elección, abre la posibilidad para poder elegir lo contrario. Dicha elección se realiza cada vez que se lleva a cabo una acción política. De este momento ético surge la visión de la democracia como una construcción permanente. "Mientras haya un nuevo hombre que se inserte a la acción política, mientras se realice una nueva acción política, aunque sea por un viejo político, la democracia siempre estará abierta, porque ese nuevo hombre o ese viejo político la está actualizando". ${ }^{37}$

Por lo tanto la sociedad del valor como la hemos llamado, o la tradicional sociedad democrática como se le ha conocido desde hace mucho, no es una sociedad a la que se pueda llegar de una vez para siempre, sino que es una forma de vida que cada momento se actualiza. Así como el valor no surge como consecuencia inmediata de la información y el conocimiento, así tampoco la sociedad del valor surge de la sociedad de la información o del conocimiento.

\section{CONCLUSIONES}

El análisis realizado nos permite concluir que aunque existe una semejanza entre los conceptos de información, conocimiento y valor en cuanto los tres son objetos ideales, al mismo tiempo se descubre una diferencia esencial entre ellos. Podemos encontrar una idea similar en Savater, quien menciona tres niveles de entendimiento de la realidad humana. El primero incluye la información que presenta los hechos y los mecanismos primarios de los hechos; el segundo consiste en el conocimiento que reflexiona sobre la información, jerarquiza su importancia y los principios generales para ordenarla; y el tercero que lleva a la sabiduría, la cual incorpora a la realidad todo lo que sabemos con el fin de encontrar una existencia mejor. ${ }^{38}$

Así pues, las tres realidades estudiadas no tienen una relación secuencial directa, inmediata y necesaria, sino son etapas que requieren diferentes fuentes y actividades. Se puede detener el camino en una de ellas y no necesariamente pasar por las tres. En el caso de la información, su fuente son los datos y la actividad requerida es la estructuración e interpretación de los mismos. Con respecto al conocimiento, su origen está

${ }^{37}$ RENDÓN ROJAS, Miguel Ángel. "Democracia: Identidad y diferencia”. En Metapolítica. V. 1. N. 2. Abril-Junio de 1997. pp. $277-$ 282. México: BUAP, CEPCOM, 1997. p. 278.

38 SAVATER, Fernando. Las preguntas de la vida. Barcelona: Ariel, 1999. p. 21. 


\section{Miguel Ángel Rendón Rojas}

dado en la información y posteriormente se requiere realizar una actividad complementaria que comprende el análisis, la síntesis, aplicar una visión dialéctica a lo que se aprende, sacar inferencias de lo aprendido, aplicar, evaluar y asimilar. Por último, el valor surge a partir de informaciones y conocimientos recibidos, pero que adicionalmente se exige una interiorización y apropiación movidos por un acto de la voluntad que elige como deseable esos principios como elementos de su proyecto existencial personal para que sirvan como guías en la interacción con la realidad.

De manera semejante, la relación entre la sociedad de la información, la sociedad del conocimiento y la sociedad del valor no es lineal. Esta última, donde se debería dar un estado de justicia, democracia, progreso, armonía y paz, no es el resultado inevitable del desarrollo del conocimiento y de la tecnología. Ya Rousseau había advertido en el Siglo de las Luces que el conocimiento no había contribuido en nada a la felicidad del hombre. Ésta es una continua búsqueda y construcción que se va actualizando día a día con la re-creación de valores, elegidos libremente por los individuos y que por consecuencia, pueden al mismo tiempo, con palabras de Dostoievsky, elegir el "ideal de la Santa Virgen o el ideal de Sodoma”.

Como profesionales de la información documental es posible e indispensable actuar en el ámbito del flujo de la información, ser colegas en la construcción del conocimiento; y como seres humanos y ciudadanos, contribuir a la actualización de una sociedad donde el motor del desarrollo sea el respeto a la dignidad y al ser del ser humano.

Artigo recebido em 02/11/2005 e aceito para publicação em 05/01/2006.

\section{NOTAS E REFERÊNCIAS}

1. Algunas propuestas de objetos de estudio de la bibliotecología son la biblioteca, la clasificación, el documento, la información, el fenómeno comunicacional, la actividad bibliotecaria, el sistema de información documental entre otros. Cf. MARTÍNEZ RÍDER, Rosa María; RENDÓN ROJAS, Miguel Ángel. Algunas propuestas latinoamericanas de objeto de estudio para la investigación bibliotecológica. Revista Interamericana de Bibliotecología, Medellín, Colombia: Universidad de Antioquia, Escuela Interamericana de Bibliotecología, v. 27, n. 1, p. 13-44, enero-jun. 2004.

2. GÓMEZ-PANTOJA, Aurora Fernandéz. Bases teóricas para el desarrollo de un código de ética para la profesión bibliotecaria. Boletín de la ANABAD, Madrid: ANABAD, n. 3, p. 15-35, jul./sept. 2001. PÉREZ PULIDO, Margarita. Códigos de ética de los bibliotecarios y otros profesionales de la información: comentario y análisis comparativo. Boletín de la ANABAD, Madrid: ANABAD, n. 3, p. 37 57, jul./sept. 2001.
3. RENDÓN ROJAS, Miguel Ángel. Bases teóricas y filosóficas de la bibliotecología. México: CUIB/UNAM, 1998. p. 62.

4. Según Locke las cualidades primarias son inseparables de los cuerpos y se captan de manera inmediata como son la extensión o la figura; las cualidades secundarias no están presentes de manera actual en los materiales para después aparecer como ente ideal; el conocimiento retoma ese ente ideal para construirse. Una distinción semejante la encontramos en Shera, quien expresa: "distinguimos entre información y conocimiento [...] la información es el insumo del conocimiento, y siempre es recibida a través de los sentidos [...] no puede haber conocimiento sin un conocedor.

5. SHERA, Jesse H. Los fundamentos de la educación bibliotecológica. México: CUIB/UNAM, 1990. p. 118. La semejanza con nuestro pensamiento es sólo en lo relativo a que la información es insumo del conocimiento, pero existe una diferencia en cuanto que para nosotros la información no se capta por los sentidos.

6. GÓMEZ DE SILVA, Guido. Breve diccionario etimológico de la lengua española. México: El Colegio de México, Fondo de Cultura Económica, 1988. p. 377.

7. RENDÓN ROJAS, Miguel Ángel. Op. Cit. p. 64.

8. Cf. PIAGET, Jean. Lógica formal y psicología genética. In: Estudios sobre lógica y psicología. Madrid: Alianza Editorial, 1982. p. 149-175. PIAGET, J. El nacimiento de la inteligencia en el niño. México: Grijalvo, 1990. PIAGET, J. Estudios de Psicología genética. Buenos Aires: Emece, 1973.

9. Cf. BEUCHOT, Mauricio. La filosofía del lenguaje en la Edad Media. 2. ed. México: UNAM, Instituto de Investigaciones Filológicas, 1991. p. 50 .

10. ECO, U. Tratado de semiótica general. Barcelona: Ed. Lumen, 1988. p. 42 .

11. Las inferencias deductivas tienen lugar cuando existe la relación de consecuencia lógica entre un juicio o enunciado, llamado conclusión, y otros juicios o enunciados, llamados premisas. La consecuencia lógica se da cuando es imposible que las premisas sean verdaderas y al mismo tiempo la conclusión sea falsa, esto es, si las premisas son verdaderas, necesariamente la conclusión será verdadera.

12. Las inferencias inductivas consisten en obtener una conclusión con base en generalizaciones de casos particulares. Su estructura general es: $a 1$ es $P, a 2$ es $P, a 3$ es $P, \ldots$, an es $P$, por lo tanto toda $a$ es $P$. No hay consecuencia lógica porque nada impida que la siguiente $a n+1$ no sea $P$, convirtiendo a la conclusión en falsa, por lo que se habla sólo de verosimilitud en los razonamientos inductivos.

13. La abducción o inferencia hipotética fue propuesta por Peirce y es una conjetura o hipótesis que se propone para resolver un problema teórico. Cf. FANN, K. T. Peirce's theory of abduction. [S.1.]: The Hague, Martinus Nijhoff, 1970. MAGNANI, Lorenzo. Abduction, reason, and science: processes of discovery and explanation. New York: Kluwer Academic/Plenum, 2001.

14. Las inferencias hermenéuticas consisten en encontrar nuevos sentidos y significados, gracias a un horizonte hermenéutico más amplio que permite "contemplar" la realidad desde otra perspectiva.

15. Cf. TERRICABRAS, Josep-Maria. Atrévete a pensar: la utilidad del pensamiento riguroso en la vida cotidiana. Barcelona: Paidos, 1999.

16. PIAGET, Jean. La epistemología genética. Madrid: Debate, 1986. p. 154-155.

17. Ibidem. p. 41-90.

18. VILlORO, Luis. Creer, saber, conocer. México: Siglo XXI, 1996. 
19. "Decir del no ser que es y decir del ser que no es, es falso. Decir del no ser que no es y del ser que es, es verdadero". ARISTÓTELES. Aristotelis metaphysica = Metafísica de Aristóteles. Ed. trilingue por Valentín García Yebra. Madrid: Gredos, 1982. 1011b.

20. RENDÓN ROJAS, Miguel Ángel. La información como ente ideal objetivizado. Investigación bibliotecológica: Archivonomía, Bibliotecología e Información. México: CUIB/UNAM, v. 9, n. 18, p. 17-24, enero/jun. 1995

21. Shera utiliza una expresión de Helprin, donde dice: "El conocimiento es información subjetiva". SHERA, J. Op. cit. p. 128.

22. GORKOVA, V. I.; GUSIEVA, T. I. Análisis de los flujos de documentación e información y estudio de las solicitudes de los usuarios de información. In: SEMINARIO INTRODUCCIÓN A LA INFORMETRÍA. ANÁLISIS DE LOS FLUJOS INFORMACIONALES Y EVALUACIÓN DE LAS FUENTES DE INFORMACIÓN, 1988, La Habana. Anales... La Habana: IDIT/ SOCICT-CI, 1988. p. 25-36.

23. MIJAILOV, A. I.; CHERNII, A. I.; GUILIAREVSKII, R. S. Fundamentos de la informática. La Habana: Nauka, Academia de Ciencias de Cuba, Instituto de Documentación e Información Científica y Técnica, 1973. p. 59-60. t. 1.

24. Cf. GORBEA PORTAL, Salvador. Producción y comunicación cientifica latinoamericana en ciencias bibliotecológica y de la información. 2004. Tesis (Doctoral) - Universidad Carlos III de Madrid, Facultad de Humanidades, Comunicación y Documentación, Getafe, 2004. p. 22.

25. RENDÓN ROJAS, Miguel Ángel. Un análisis del concepto sociedad de la información desde el enfoque histórico. Información, Cultura y Sociedad, Buenos Aires: Universidad de Buenos Aires, n. 4, p. 9-22, p. 16, 2001.

26. Cf. RENDÓN ROJAS, Miguel Ángel. Sociedad del conocimiento. In: ALMADA DE ASCENCIO, Margarita et al. (Ed.). Contribución al desarrollo de la sociedad del conocimiento. México: CUIB/UNAM, [s. d.]. p. 50-57.
27. Ibídem. p. 150-151.

28. VOLTAIRE. Tratado sobre la tolerancia. Madrid: Santillana, 1997. p. 29.

29. KANT, Inmanuel. Crítica de la razón práctica. México: UAMI, 2001.

30. GADAMER, Hans-Georg. Verdad y método I: fundamentos de una hermenéutica filosófica. Salamanca: Sígueme, 1993. p. 41.

31. Ibídem. p. 40.

32. KANT, Inmanuel. Pedagogía. Madrid: Akal, 1983. p. 80-81.

33. DOSTOIEVSKY, Fiódor. Mijaílovich: dnievnik pisatelia (Diario del escritor). In: DOSTOIEVSKY o russkoi literaturi (Dostoievsky sobre la literatura rusa). Moscú: [s.n.], 1987. p. 267.

34. Es sintomático que por ejemplo el creador del Gulag se haya hecho llamar Stalin, que proviene de la palabra rusa stal, que significa acero.

35. FERRARIS, Mauricio. La hermenéutica. México: Taurus, 2001. p. 51.

36. Cfr. SARTORI, G. Democrazia e definizioni, Bologna: Il Molino, 1969. cap. V. También véase: CANSINO, César. La democracia entre utopía y realidad. Universidad de México, México: UNAM, n. 540, p. 66-68, enero 1996. RENDÓN ROJAS, Miguel Ángel. Democracia: identidad y diferencia. Metapolítica, México: BUAP, v. 1, n. 2, p. 277 282, abr./jun. 1997.

37. RENDÓN ROJAS, Miguel Ángel. Democracia: identidad y diferencia. Metapolítica, México: BUAP, v. 1, n. 2, p. 277-282, p. 278, abr.jun. 1997.

38. SAVATER, Fernando. Las preguntas de la vida. Barcelona: Ariel, 1999. p. 21. 